\begin{tabular}{|c|l|}
\hline Title & Inactivation of the central thal amus delays self-timed saccades. \\
\hline Author(s) & Tanaka, Masaki \\
\hline Citation & $\begin{array}{l}\text { Nature Neuroscience, 9(1), 20-22 } \\
\text { https://doi.org/10.1038/hn1617 }\end{array}$ \\
\hline Issue Date & 2006-01 \\
\hline Doc URL & http://hdl.handle.net/2115/43045 \\
\hline Type & article (author version) \\
\hline File Information & NatureNeuro9.pdf \\
\hline
\end{tabular}

Instructions for use 


\title{
Inactivation of the central thalamus delays self-timed saccades
}

\author{
Masaki Tanaka \\ Department of Physiology, Hokkaido University School of Medicine, Sapporo 060-8638, Japan
}

Manuscript ID: NN-BC15392A

Number of figures: 3

Abstract: 62 words

Text, Figure legends, and References: 2087 words

Number of References: 15

\author{
Correspondence to: \\ Masaki Tanaka \\ Department of Physiology \\ Hokkaido University School of Medicine \\ North 15, West 7, Sapporo 060-8638, JAPAN \\ Tel: $\quad+81117065040$ \\ FAX: +81117065041 \\ E-mail: masaki@med.hokudai.ac.jp
}




\section{ABSTRACT}

The central thalamus transmits corollary discharge signals for eye movement control, but its role in eye movement generation remains uncertain. Inactivation of the paralaminar part of the ventrolateral thalamus delayed the initiation of contraversive saccades, particularly during a novel memory-guided saccade task that required self-triggering of the movement. The results suggest that signals through the thalamus regulate the timing of self-initiated saccades. 
Although signals through the motor thalamus play crucial roles in the generation of somatic movements ${ }^{1}$, their roles in eye movement generation remain unknown. Previous studies have demonstrated that neurons in the paralaminar part of the ventrolateral thalamus modulate their activity during the preparation as well as the execution of saccades $^{2-4}$. To investigate possible roles of these signals in saccade generation, the present study examined effects of local inactivation. All experimental protocols were approved in advance by the Animal Care and Use Committee of the Hokkaido University School of Medicine, and were in accordance with the NIH Guide for the Care and Use of Laboratory Animals.

Monkeys $(n=3)$ were trained to perform three saccade paradigms. In the visually-guided saccade paradigm (Fig. 1a, left), a central fixation target was extinguished at the time of peripheral target onset, and monkeys were required to make a saccade to the target within $400 \mathrm{~ms}$. In the memory-guided saccade paradigm (Fig. 1a, middle), a peripheral target ('cue') was flashed briefly (200 ms) during fixation. Monkeys were required to maintain fixation for an additional 1,000-1,200 ms, and make a saccade to the cue location immediately after the offset of the fixation target (Supplementary Fig. 1). Because of a delay between offsets of the cue and fixation target, the direction and amplitude of saccades must be programmed based on internal signals that are related to the cue location. In this paradigm, however, the time of saccade initiation was instructed externally by the offset of the fixation target. The paradigm therefore will be referred to as an 'externally triggered' memory-guided saccade paradigm in this study; this is essentially identical to classic memory-guided saccade tasks in the literature $e^{2,3,5}$.

A novel version of the memory-guided saccade paradigm required self-timing (Fig. 1a, right). It began like the classic memory-guided saccade task, but no overt instruction to move was given; instead, monkeys were required to make a saccade to the cue location $800-1,600 \mathrm{~ms}$ after the offset of the cue. The fixation target was extinguished immediately after initiation of the saccade, and the peripheral target reappeared $400 \mathrm{~ms}$ later and remained visible for an additional $800 \mathrm{~ms}$ 
(Supplementary Fig. 1). Monkeys were rewarded only when they generated a saccade within an 800 -ms time window and when their eye position was within $5^{\circ}$ from the peripheral target as long as it was visible. In this 'internally-triggered' memory-guided saccade paradigm, both the timing and metrics of saccades must be programmed based on internal signals. The location of the saccade target was either $12^{\circ}$ right or left of the central fixation and a total of six different trials ( 3 paradigms in 2 directions) were presented in random order within a block.

To examine whether signals in the central thalamus contribute to saccade generation, a small amount of GABA agonist (muscimol hydrobromide; $5 \mu \mathrm{g} / \mu \mathrm{l}$ in saline; volumes ranging from 1-2 $\mu 1$, mean volume 1.6 $\pm 0.3 \mu 1, n=47$ ) was locally injected (Fig. 1b, see Supplementary Methods for the details). Inactivation sites were selected as those exhibiting eye movement-related neuronal activities in separate recording experiments ${ }^{6}$. Fig. 2a plots the cumulative relative distribution of individual saccade latencies in a single experimental session. For presentation purposes only, the scale on the abscissa in the right panels is three times greater than those in the other panels. The monkey generated saccades within a 400 or 800 -ms time window (horizontal bar in each panel) in most trials, both before and after inactivation. However, following injection of muscimol, data for contraversive saccades became more variable, and tended toward longer latencies that were statistically different from the values obtained before injection (Fig. 2a, top panels, Wilcoxon rank-sum test, $P<0.01$ ). On the other hand, the latency of ipsiversive saccades was shortened slightly in the internally triggered memory-guided saccade task $(P<0.01)$, but was not altered in the other two tasks (Fig. 2a, bottom). Inactivation effects were also evident in both the accuracy and dynamics of saccades. Fig. $2 \mathbf{b}$ plots individual saccade endpoints before (black dots) and after (colored dots) inactivation. After inactivation, the distance between target and saccade endpoints increased significantly for all contraversive saccades and ipsiversive externally triggered memory-guided saccades (two-tailed t-test, $P<0.01)$, but not for the others $(P>0.05)$. This was primarily due to increased scatter, and not to systematic changes in mean saccade amplitude that ranged from -0.52 to $0.72^{\circ}$. The peak velocity 
of individual saccades decreased slightly but significantly for contaversive saccades and ipsiversive internally triggered memory-guided saccades (two-tailed t-test, $P<0.01$ ). The data from individual injection sites are summarized in Supplementary Fig. 2, Supplementary Fig. 3 and Supplementary Fig. 4 for the accuracy, amplitude, and velocity of saccades, respectively.

Similar effects of inactivation were found for multiple sites in four thalami of the three monkeys. Among 47 sites tested, inactivation at 30 sites significantly altered the latency of saccades in either or both directions (Wilcoxon rank-sum test, $P<0.01$ ). The amounts of muscimol injected into the effective and non-effective sites were not statistically different (two-tailed t-test, $P=0.86$ ). Fig. 3a compares medians of saccade latencies before and after injection for 47 muscimol (circles) and 7 saline control sites (crosses). As previously, scales for the internally triggered task are four times greater than those for the two other tasks. In the internally triggered task, thalamic inactivation effectively lengthened the latency of contraversive saccades (Wilcoxon rank-sum test, $P<0.01$ ) for 26 out of 47 sites, whereas the latency of ipsiversive saccades was unchanged (17 sites) or even decreased ( 9 sites, $P<0.01$ ). In the other tasks, the effect of inactivation was much smaller, but was statistically significant for contraversive saccades in most sites (23 and 19 sites for the externally triggered task and the visually-guided task, respectively) and for ipsiversive saccades in some sites (7 and 4 sites). Fig. 3b summarizes means and 95\% confidence intervals of the changes in median latency for different conditions. The effect on latency was greater for contraversive saccades, and was greatest for the internally triggered memory-guided saccades. In all three paradigms, differences in median latencies were statistically different from zero for contraversive saccades (two-tailed t-test, $P$ $\left.<10^{-5}\right)$, but were not for ipsiversive saccades $(P>0.05)$. Injection of saline into the effective sites $(n$ $=7$, mean volume, $1.8 \pm 0.2 \mu \mathrm{l}$ ) did not result in a significant change in latency for all six paradigms $(P>0.1)$. A one-way analysis of variance (ANOVA) showed that the changes in median latencies following muscimol injection were significantly different across paradigms for contraversive saccades $(P<0.01)$ but not for ipsiversive saccades $(P=0.09)$. For contraversive saccades, a post-hoc 
comparison (Scheffe) revealed that the values for the internally triggered task were statistically different from those for any of the other two tasks $\left(P<10^{-5}\right)$, but that the values for the other two tasks were not different from each other $(P>0.05)$. Thus, thalamic inactivation did not produce a fixed shift in saccade timing, but showed more profound effects on self-timed, contraversive saccades.

The present results showed that inactivation of the rostral portion of the caudal division of the ventrolateral nucleus and the adjacent area $\mathrm{X}$ of the thalamus altered the latency of contraversive saccades. This study also confirmed the previous findings that inactivation of the mediodorsal thalamus caused negligible deficits in saccade generation ${ }^{7}$ (Fig. 1b). The inactivation effects of the ventrolateral thalamus were much more profound in tasks that required monkeys to internally determine both goals and timing of saccades. These findings suggest new insights into the roles of the central thalamus in eye movement control. Previous studies showed that signals in the central thalamus are used for internal monitoring of eye movements ${ }^{7}$, but that they do not contribute to the initiation of visually-guided eye movements ${ }^{6,7}$ (Supplementary Fig. 5 plots injection sites in this and the previous study that examined effects on smooth pursuit ${ }^{6}$ ). Other studies suggested that signals in the ventrolateral ${ }^{8}$ and mediodorsal ${ }^{9}$ thalamus link spatial information about target location and saccade goals. The present data imply an additional role of the central thalamus, one that regulates the timing of self-initiated saccades.

The central thalamus plays a pivotal role in regulating the activity in both the cerebral cortex and the basal ganglia. Given that the diameter of muscimol spread could be up to a few millimeters ${ }^{10}$, the present experiments likely disrupted signals in multiple thalamic pathways. Inactivation of the ventrolateral thalamus and the adjacent rostral intralaminar nuclei could modulate neuronal processes within the reciprocal thalamocortical loops ${ }^{11}$ and/or the local basal ganglia-thalamostriatal circuitry ${ }^{12}$. In addition, thalamic inactivation could also disturb signals from the basal ganglia and cerebellum to the cerebral cortex ${ }^{13}$. Since previous studies suggested that the latter pathways are involved in timing of somatic movements and time perception ${ }^{14,15}$, the delay of self-timed saccades possibly resulted 
from inactivation of these pathways. The signals through the thalamus to the cortex might regulate the timing of decisions for internally triggered movements, including saccades. 


\section{ACKNOWLEDGEMENTS}

I thank O. Hikosaka for comments on a previous version of the manuscript; M. Takada for identification of the thalamic nuclei on histological sections; S. Hirano and A. Yoshida for technical assistance; M. Suzuki for administrative help. One monkey was provided by the Primate Research Institute of the Kyoto University. This work was supported by a grant-in-aid for scientific research on priority area (16015204, 17022003) and a grant for young scientists (B17700363) from the Ministry of Education, Culture, Sports and Technology of Japan.

Competing interests statement: The author declares that he has no competing financial interests. 


\section{REFERENCES}

1. van Donkelaar, P., Stein, J.F., Passingham, R.E. \& Miall, R.C. J. Neurophysiol. 83, 2780-2790 (2000).

2. Wyder, M.T., Massoglia, D.P. \& Stanford T.R. J. Neurophysiol. 90,2029-2052 (2003).

3. Tanibuchi, I. \& Goldman-Rakic, P.S. J. Neurophysiol. 93, 614-619 (2005).

4. Schlag-Rey, M. \& Schlag, J. J. Neurophysiol. 51, 1149-1174 (1984).

5. Hikosaka, O. \& Wurtz, R.H. J. Neurophysiol. 49, 1268-1284 (1983).

6. Tanaka, M. J. Neurosci. 25, 5866-5876 (2005).

7. Sommer, M.A. \& Wurtz, R.H. Science 296, 1480-1482 (2002).

8. Wyder, M.T., Massoglia, D.P. \& Stanford, T.R. J. Neurophysiol. 91, 2628-2648 (2004).

9. Watanabe, Y. \& Funahashi, S. J. Neurophysiol. 92, 1756-1769 (2004).

10. Martin, J.H. Neurosci. Lett. 127, 160-164 (1991).

11. McFarland, N.R. \& Haber, S.N. J. Neurosci. 22, 8117-8132 (2002).

12. Smith, Y., Raju, D.V., Pare, J.F. \& Sidibe, M. Trends Neurosci. 27, 520-527 (2004)

13. Middleton, F.A. \& Strick, P.L. Brain Res. Brain Res. Rev. 31, 236-250 (2000).

14. Lewis, P.A. \& Miall, R.C. Curr. Opin. Neurobiol. 13, 250-255 (2003).

15. Ivry, R.B. \& Spencer, R.M. Curr. Opin. Neurobiol. 14, 225-232 (2004). 


\section{FIGURE LEGENDS}

Figure 1 Behavioral paradigms and injection sites. (a) Sequence of events in three paradigms. The color of the fixation point was different between two memory-guided saccade tasks to inform monkeys of the trial type. (b) Injection sites for two monkeys. The size of each symbol indicates the amount of the change in median latencies in the contraversive internally triggered task. Gray fillings indicate effective sites (Wilcoxon rank-sum test, $P<0.01$ ). The levels of frontal sections are shown as the position posterior to the anterior commissure (AC). MD, mediodorsal; VA, ventroanterior; VLc and VLo, caudal and oral divisions of ventrolateral, respectively; VPLo, oral division of ventroposterolateral; VPM, ventroposteriomedial; X, area X.

Figure 2 Data from a single experiment. (a) Cumulative relative frequency of saccade latency before (black) and after (colored) inactivation of the left thalamus. Each panel plots data of 177-206 saccades. Horizontal bar indicates the time window to obtain reward. The number on each panel indicates the difference in medians. Note that the scale on the abscissa in the right column is three times greater than that in the others. (b) Distributions of saccade endpoints before and after inactivation.

Figure 3 Summary of inactivation effects on saccade latency for multiple sites. (a) Each panel compares median latencies before and after injection. Note that scales in the right panels are four times greater than those in the others. The red symbols indicate data showing a significant inactivation effect (Wilcoxon rank-sum test, $P<0.01$ ), whereas the open symbols indicate those without it. The blue crosses plot data for seven saline controls. One outlier has been omitted from the top-right panel $([1160,1731])$. (b) Means and 95\% confidence intervals of the changes in median latencies. For some data points, the sizes of symbols are larger than the error bars. 


\section{Supplementary Figure legends}

Supplementary Figure 1. Time courses of three saccade paradigms. Horizontal gray bars indicate time intervals during which monkeys were required to make a saccade to obtain reward. In the visually-guided saccade task, the fixation point disappeared at the time of saccade target onset. In the externally triggered memory-guided saccade task, the fixation point disappeared after a random $1,000-1,200 \mathrm{~ms}$ interval following the cue offset. In the internally triggered memory-guided saccade task, the fixation point disappeared only after saccade initiation within an 800-ms time window.

Supplementary Figure 2. Effects of inactivation on the accuracy of saccades. Each panel compares means of distances from target location to saccade endpoints before and after inactivation. The red symbols indicate data showing significant inactivation effects (two-tailed t-test, $P<0.01$ ), whereas open symbols indicate data without a significant difference $(P>0.01)$. The blue crosses plot data from seven saline control experiments. In the population, thalamic inactivation slightly but significantly altered saccade accuracy in the internally triggered memory-guided tasks in both directions and the contraversive externally triggered memory-guided task (two-tailed t-test, $P<0.01$, $n=47)$. Injection of saline vehicle did not alter saccade accuracy in all six paradigms $(P>0.2, n=7)$. The difference in means before and after inactivation averaged $0.03 \pm 0.18,0.53 \pm 0.37$ and $0.63 \pm$ $0.39^{\circ}$ for contraversive saccades in the visual, external and internal tasks, respectively, and $0.02 \pm$ $0.17,0.14 \pm 0.29$ and $0.18 \pm 0.25^{\circ}$ for ipsiversive saccades, respectively. A one-way ANOVA showed that the changes in saccade accuracy were different across paradigms in both directions $(P<0.01)$. For contraversive saccades, a multiple comparison (Scheffe) revealed a significant difference $(P<$ 0.01) between the visually-guided task and each of the other tasks but not between the two memory-guided tasks. For ipsiversive saccades, a significant difference was detected between the internally triggered task and the visually-guided task but not for the other two pairs. 
Supplementary Figure 3. Effects of inactivation on the amplitude of saccades. The graph format is same as for Fig. 3a and Supplementary Fig. 2. The red symbols indicate data showing significant inactivation effects (two-tailed t-test, $P<0.01$ ), whereas open symbols indicate data without a significant difference $(P>0.01)$. The blue crosses plot data from seven saline control experiments. In the population, thalamic inactivation significantly altered saccade amplitude in the contraversive memory-guided tasks (two-tailed t-test, $P<0.01, n=47)$, but not in other four tasks $(P>0.05)$. Saccade amplitude was not altered following saline injection in all six paradigms $(P>0.05, n=7)$. The difference in means before and after inactivation averaged $-0.03 \pm 0.24,0.41 \pm 0.48$ and $0.54 \pm$ $0.57^{\circ}$ for contraversive saccades in the visual, external and internal tasks, respectively, and $0.07 \pm$ $0.31,0.23 \pm 0.43$ and $0.22 \pm 0.40^{\circ}$ for ipsiversive saccades, respectively. A one-way ANOVA showed that the changes in saccade accuracy were different across paradigms for contraversive saccades $(P<$ 0.05) but not for ipsiversive saccades. For contraversive saccades, a post-hoc comparison (Scheffe) detected a significant difference between the visually-guided saccades and each of the memory-guided saccades $(P>0.05)$.

Supplementary Figure 4. Effects of inactivation on saccade velocity. The graph format is same as for Fig. 3a and Supplementary Figs. 2 and 3. The red symbols plot data showing a significant inactivation effect (two tailed t-test, $P<0.01$ ), whereas the open symbols indicate data without it. Blue crosses indicate data for saline control experiments. In the population, thalamic inactivation significantly altered velocity of contraversive memory-guided saccades (two-tailed t-test, $P<0.01, n$ $=47)$, but not in other four tasks. Injection of saline into the effective sites did not alter saccade velocity in all six paradigms $(P>0.5, n=7)$. The changes in saccade velocity after inactivation averaged $-9.4 \pm 17.8,-42.7 \pm 55.8$ and $-38.3 \pm 58.4^{\circ} \mathrm{s}^{-1}$ for contraversive saccades, and $-2.6 \pm 17.4$, $13.7 \pm 32.7$ and $16.6 \pm 36.7^{\circ} \mathrm{s}^{-1}$ for ipsiversive saccades. The amount of changes in contraversive saccade velocity was statistically different across paradigms (one-way ANOVA, $P<0.01$ ). A 
post-hoc comparison (Scheffe) detected a significant difference $(P<0.01)$ between the visually-guided saccades and externally triggered memory-guided saccades, but not for the other two pairs.

Supplementary Figure 5. Comparison of injection sites that affected saccades and smooth pursuit. The symbols in different colors indicate different effects on eye movements. Although inactivation at many sites altered both types of eye movements, there were some sites where inactivation modified either saccades or pursuit exclusively. 

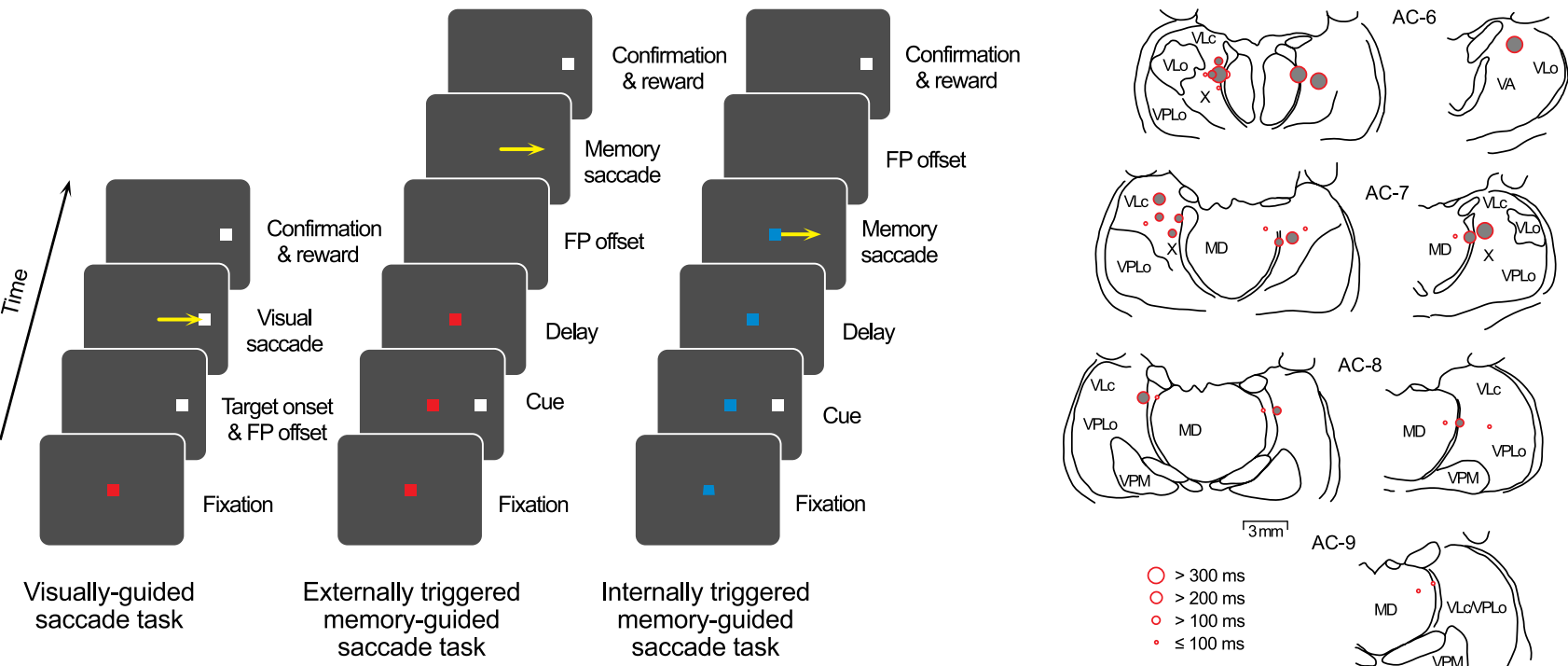

$$
\begin{aligned}
& O>300 \mathrm{~ms} \\
& \mathrm{O}>200 \mathrm{~ms} \\
& \circ>100 \mathrm{~ms} \\
& \cdot \leq 100 \mathrm{~ms}
\end{aligned}
$$

AC- 9

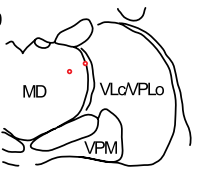




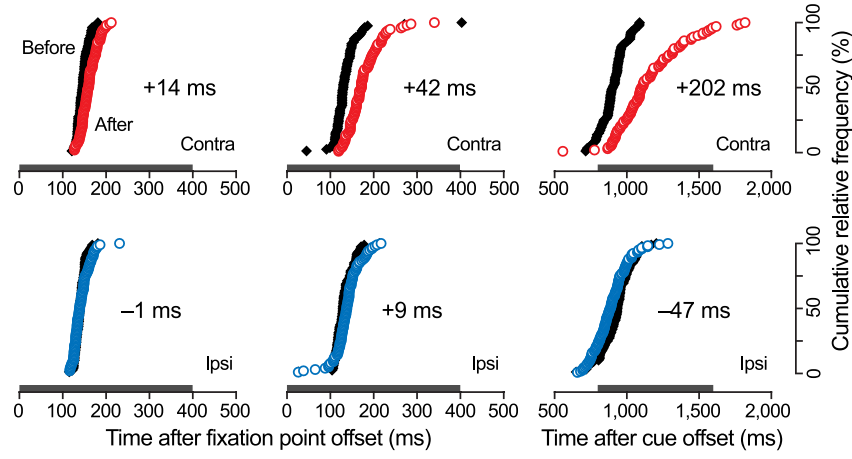

b Visual

$-16-12-8$

\section{External}

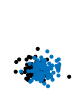

Internal

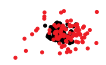

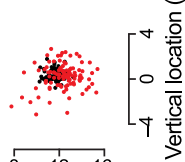

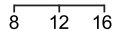
Horizontal location of saccade endpoint $\left({ }^{\circ}\right)$ 


\section{a Visual}
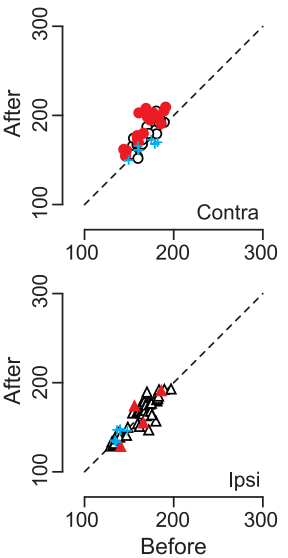

External
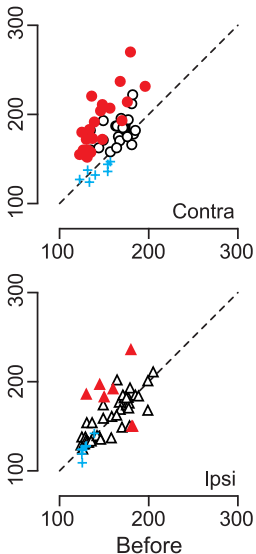

Time after fixation point offset (ms)
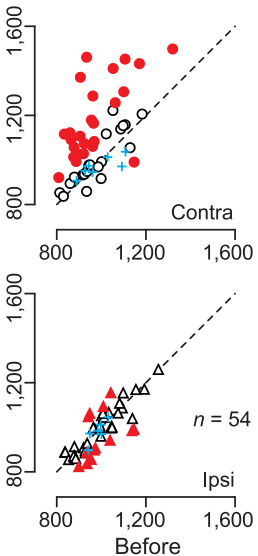

Time after cue offset (ms)
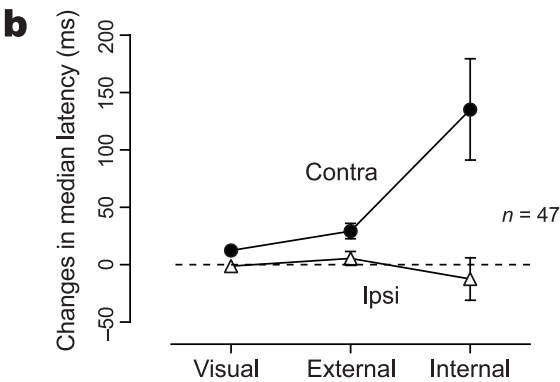

fig. 3 tanaka 


\section{Supplementary Methods}

Animal preparation. Three Japanese monkeys (Macaca fuscata, 6-12 kg) were used. All experimental protocols were approved in advance by the Animal Care and Use Committee of the Hokkaido University School of Medicine, and were in accordance with the NIH Guide for the Care and Use of Laboratory Animals (National Research Council, 1996). Animals were prepared for chronic experiments by implanting a head-holding device and eye coil under general halothane and pentobarbital anesthesia and using sterile procedures. During experiments and training sessions, the monkey's head was secured to a primate chair, and horizontal and vertical eye movements were recorded using the search coil technique. After training on oculomotor tasks, another surgery was performed to implant a recording cylinder over a small craniotomy that was placed at A10-13, L5. The location of cylinder was somewhat anterior to the previous studies that used rhesus monkeys ${ }^{1-4}$, since the stereotaxic coordinates of the motor thalamus extend more anteriorly in Japanese monkeys ${ }^{5}$. Animals received analgesia with either suppository acetaminophen or intramuscular injection of pentazocine after each surgery. Topical antibiotics were administered around the implant and in the cylinder as necessary. Water intake of monkeys was controlled daily so that they were motivated to perform oculomotor tasks.

Stimuli and procedures. Experiments were controlled by a Windows-based real-time data acquisition system (TEMPO, Reflective Computing). All events were updated every $5 \mathrm{~ms}$, and visual stimuli were presented on a 24-inch cathode-ray tube monitor (SONY, GDM-FW900, refresh rate: $60 \mathrm{~Hz}$ ) that was located $38 \mathrm{~cm}$ away from eyes and subtended $64 \times 44^{\circ}$ of visual angle. A $0.5^{\circ}$ square spot served as the visual stimulus. Monkeys were required to move their eyes within a 'window' that surrounded the target location at specific time intervals to obtain a water or apple juice reward. The size of the window was $2^{\circ}$ for initial fixation, and $5^{\circ}$ for the peripheral target. 
Since previous studies show no clear topography of eye movement signals in the ventrolateral thalamus ${ }^{2,6}$, the peripheral target was placed either $12^{\circ}$ right or left of the central fixation throughout the experiments. In both the visually-guided saccade task and the externally triggered memory-guided task, monkeys were required to make a saccade within $400 \mathrm{~ms}$ after the offset of the fixation target (Supplementary Fig. 1). The fixation target was extinguished at the time of the peripheral target onset (visually-guided task) or within a random 1,000-1,200 ms interval following the cue offset (externally triggered memory-guided task). In the internally triggered memory-guided saccade task, the fixation target disappeared when monkeys made a saccade $800-1,600 \mathrm{~ms}$ after the cue offset. Since monkeys were sometimes unable to initiate a saccade until the end of this interval following thalamic inactivation, the fixation target was also extinguished 1,600 ms after the cue offset (without cue reappearance) so as to obtain a balanced number of data across paradigms. Monkeys did not receive a reward in such a trial even if they generated a memory-guided saccade to the previously presented cue location in response to the fixation target offset, but the identical task was not repeated within the block that included six different saccade tasks. With this exception, the trial was aborted and followed by a newly selected trial if monkeys failed to maintain their eye position within a specified window or could not generate a saccade within a specified time interval. Data of error trials were also saved as appropriate files and were analyzed off-line (see below).

Prior to the inactivation experiments described here, single and multiple unit activities were recorded from the central thalamus when monkeys performed a variety of eye movement tasks in separate experiments ${ }^{7}$. Sites of inactivation were within the area containing neurons that modulated the activity during saccades, the delay period, or smooth pursuit. To induce reversible inactivation in the central thalamus, a small amount of muscimol was pressure injected at a rate of about $0.2 \mu 1$ per $30 \mathrm{~s}$ using a 30-gauge injection needle that was connected to a micro-syringe and was penetrated through a 23-gauge guide tube. Site and depth of injection were adjusted by using an $\mathrm{X}-\mathrm{Y}$ stage attached on top of the cylinder and a micromanipulator (Narishige, MO-97S) that was 
also used for the unit recording experiments. The effect of inactivation was assessed by comparing data obtained before and 10-90 min after muscimol injection. A previous study showed that the spread of muscimol $(1 \mu \mathrm{g}$ in $1 \mu \mathrm{l})$ injected into the rat cerebral cortex was about $1.7 \mathrm{~mm}$, and that the width of spread remained relatively constant for 30-120 min after injection ${ }^{8}$. The inactivation effects persisted for the period of the experiments, but disappeared the next day. When medians of saccade latency before inactivation at effective sites were compared with those obtained the next day, the values were not significantly different for all three paradigms in both directions (two-tailed t-test, $P>0.1, n=9)$. Among 30 sites where inactivation modified saccade latency, inactivation at 17 sites also altered smooth pursuit velocity ${ }^{7}$. To allow for comparisons with the sites where inactivation altered pursuit, Supplementary Fig. 5 plots injection sites analyzed in this and the previous study. These injection sites in two monkeys were reconstructed from histological sections based on stereotaxic coordinates and locations of several electrolytic lesions that were made at the end of experiments. Histology from the third animal was not obtained because he is still in use for another experiment. However, the similar inactivation effects between monkeys and the existence of eye movement-related neuronal activities indicated that the sites of inactivation in this monkey were comparable to those in the others. The nomenclature of the thalamic nuclei was after Olszewski ${ }^{9}$.

Data acquisition and analysis. Horizontal and vertical eye position signals were obtained directly from eye coil electronics (Enzanshi Kogyo, MEL-25). Data were digitized and sampled at 1 $\mathrm{kHz}$ during experiments, and stored in files with other codes of the task event and the vertical retrace signals for subsequent off-line analysis that was performed using Matlab (Mathworks). Data were not collected during the intertrial intervals (500-800 ms). Eye velocity was obtained by digital differentiation, and each saccade was detected when angular eye velocity exceeded $40^{\circ} \mathrm{s}^{-1}$. Data were included for further analysis if saccade was generated within 500-2,000 ms following the cue 
offset (internally triggered task) or within $600 \mathrm{~ms}$ following the fixation target offset (other tasks), and if saccade amplitude was greater than $6^{\circ}$ and the endpoint of saccade was within $5^{\circ}$ from the peripheral target. Since the fixation target disappeared when monkeys failed to generate a saccade within 1,600 ms after cue offset in the internally triggered memory-guided saccade task (see above), the saccades that were initiated $>1,700 \mathrm{~ms}$ after the cue offset were essentially "externally triggered' memory-guided saccades. The proportions of such trials before and after inactivation averaged 0.9 and $11.1 \%$, respectively, for contraversive saccades in experiments that were performed on 30 effective sites. Inclusion of these data would underestimate the changes in mean saccade latencies in the internally triggered memory-guided task, since monkeys would have made self-initiated saccades with longer latency in such trials if the fixation target was not extinguished $1,600 \mathrm{~ms}$ after the cue offset. Assuming that the latency of self-initiated saccades in those non-rewarded trials would be longer but not shorter than $1,700 \mathrm{~ms}$, the effects of inactivation on saccade latency were assessed by comparing the medians. In almost all experiments (46 of 47) the median latency was less than 1,700 ms even after thalamic inactivation (Fig. 3a, right). Similarly, because the peripheral target reappeared $400 \mathrm{~ms}$ after fixation target offset in the externally triggered memory-guided saccade task (Supplementary Fig. 1), the saccades that were initiated $>$ $500 \mathrm{~ms}$ after the fixation target offset would be 'visually-guided'. The proportions of such trials for contraversive saccades before and after inactivation were only $0.1 \%$ and $0.5 \%$, respectively, and the values after inactivation were not statistically greater than that before inactivation (one-tailed t-test, $P=0.10, n=30)$. The exact time of target offset was computed based on target location and the vertical retrace signal, and the time between target offset and saccade initiation was expressed as latency.

\section{References}

1. Sommer, M.A. \& Wurtz, R.H. Science 296, 1480-1482 (2002). 
2. Wyder, M.T., Massoglia, D.P. \& Stanford T.R. J. Neurophysiol. 90,2029-2052 (2003).

3. Tanibuchi, I. \& Goldman-Rakic, P.S. J. Neurophysiol. 89, 1067-1077 (2003).

4. Watanabe, Y. \& Funahashi, S. J. Neurophysiol. 92, 1756-1769 (2004).

5. Kusama, T. \& Mabuchi, M. Stereotaxic atlas of the brain of Macaca fuscata. (University of Tokyo, Tokyo, 1970).

6. Schlag-Rey, M. \& Schlag, J. J. Neurophysiol. 51, 1149-1174 (1984).

7. Tanaka, M. J. Neurosci. 25, 5866-5876 (2005).

8. Martin, J.H. Neurosci. Lett. 127, 160-164 (1991).

9. Olszewski, J. The thalamus of the Macaca mulatta: An atlas for use with the stereotaxic instrument. (Karger, Basel, Switzerland, 1952). 


\section{Visually-guided saccade task}

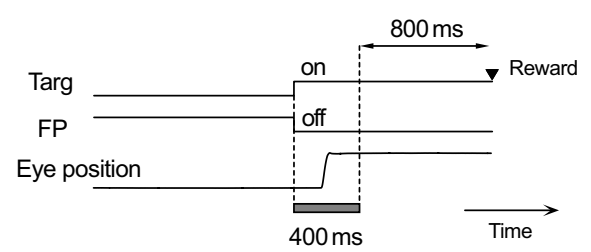

\section{Externally triggered memory-guided saccade task}

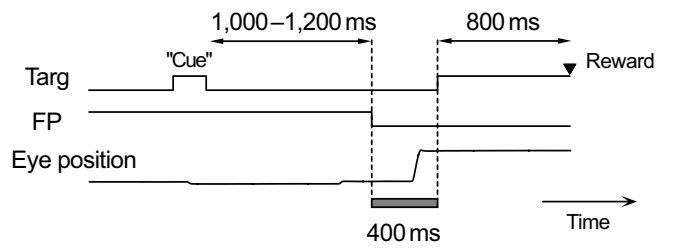

\section{Internally triggered memory-guided saccade task}

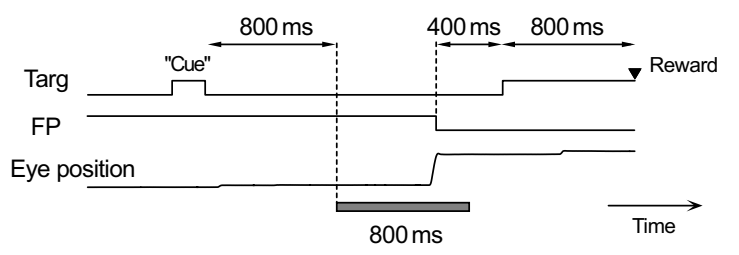

Supplementary Figure 1. Time courses of three saccade paradigms. Horizontal gray bars indicate time intervals during which monkeys were required to make a saccade to obtain reward. In the visually-guided saccade task, the fixation point disappeared at the time of saccade target onset. In the externally triggered memoryguided saccade task, the fixation point disappeared after a random 1,000-1,200 ms interval following the cue offset. In the internally triggered memory-guided saccade task, the fixation point disappeared only after saccade initiation within an 800-ms time window. 
Visual

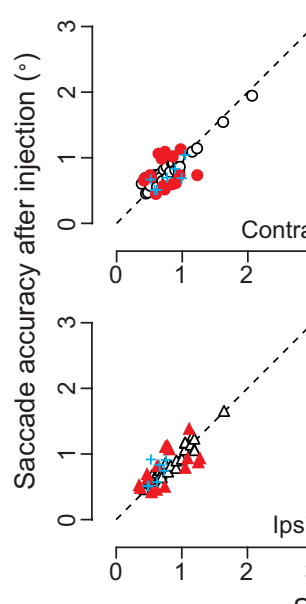

External
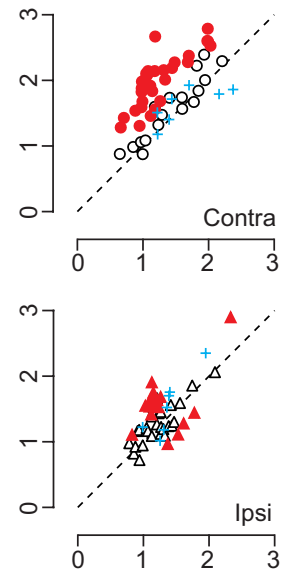

Saccade accuracy before injection $\left({ }^{\circ}\right)$

Internal
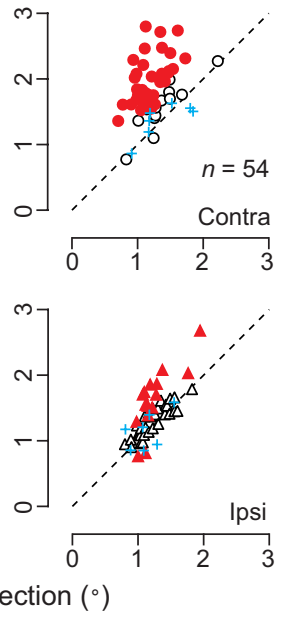

Supplementary Figure 2. Effects of inactivation on the accuracy of saccades. Each panel compares means of distances from target to saccade endpoints before and after inactivation. The red symbols indicate data showing a significant inactivation effect (two-tailed t-test, $P<0.01$ ), whereas the open symbols indicate data without a significant difference $(P>0.01)$. The blue crosses plot data from seven saline control experiments. In the population, thalamic inactivation slightly but significantly altered saccade accuracy in the internally triggered memory-guided tasks in both directions and the contraversive externally triggered memory-guided task (two-tailed t-test, $P<0.01, n=47$ ). Injection of saline vehicle did not alter saccade accuracy in all six paradigms $(P>0.2, n=7)$. The difference in means before and after inactivation averaged $0.03 \pm 0.18,0.53 \pm 0.37$ and $0.63 \pm 0.39^{\circ}$ for contraversive saccades in the visual, external and internal tasks, respectively, and $0.02 \pm 0.17,0.14 \pm 0.29$ and $0.18 \pm 0.25^{\circ}$ for ipsiversive saccades, respectively. A one-way ANOVA showed that the changes in saccade accuracy were different across paradigms in both directions $(P<0.01)$. For contraversive saccades, a multiple comparison (Scheffe) revealed a significant difference $(P<0.01)$ between the visually-guided task and each of the other tasks but not between the two memory-guided tasks. For ipsiversive saccades, a significant difference was detected between the internally triggered task and the visually-guided task but not for the other two pairs. 


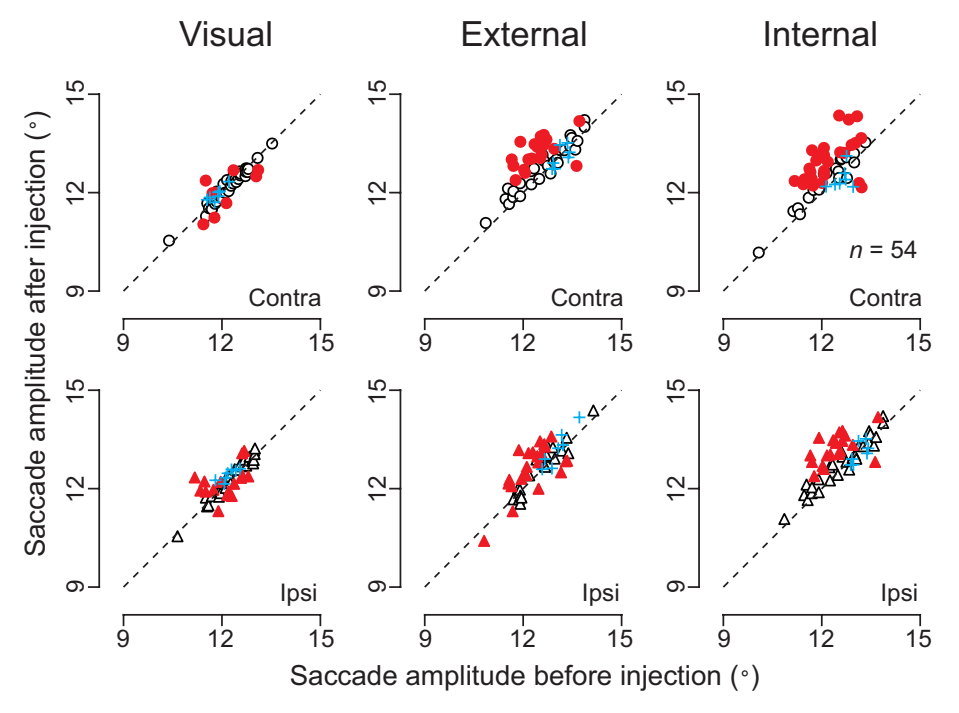

Supplementary Figure 3. Effects of inactivation on the amplitude of saccades. The graph format is same as for Fig. 3a and Supplementary Fig. 2. The red symbols plot data showing significant inactivation effects (two-tailed t-test, $P<$ $0.01)$, whereas open symbols indicate data without a significant difference $(P>$ $0.01)$. The blue crosses plot data from seven saline control experiments. In the population, thalamic inactivation significantly altered saccade amplitude in the contraversive memory-guided tasks (two-tailed t-test, $P<0.01, n=47$ ), but not in other four tasks $(P>0.05)$. Saccade amplitude was not altered following saline injection in all six paradigms $(P>0.05, n=7)$. The difference in means before and after inactivation averaged $-0.03 \pm 0.24,0.41 \pm 0.48$ and $0.54 \pm 0.57^{\circ}$ for contraversive saccades in the visual, external and internal tasks, respectively, and $0.07 \pm 0.31,0.23 \pm 0.43$ and $0.22 \pm 0.40^{\circ}$ for ipsiversive saccades, respectively. A one-way ANOVA showed that the changes in saccade amplitude were different across paradigms for contraversive saccades $(P<0.05)$, but not for ipsiversive saccades. For contraversive saccades, a post-hoc comparison (Scheffe) detected a significant difference between the visually-guided saccades and each of the memory-guided saccades $(P>0.05)$. 


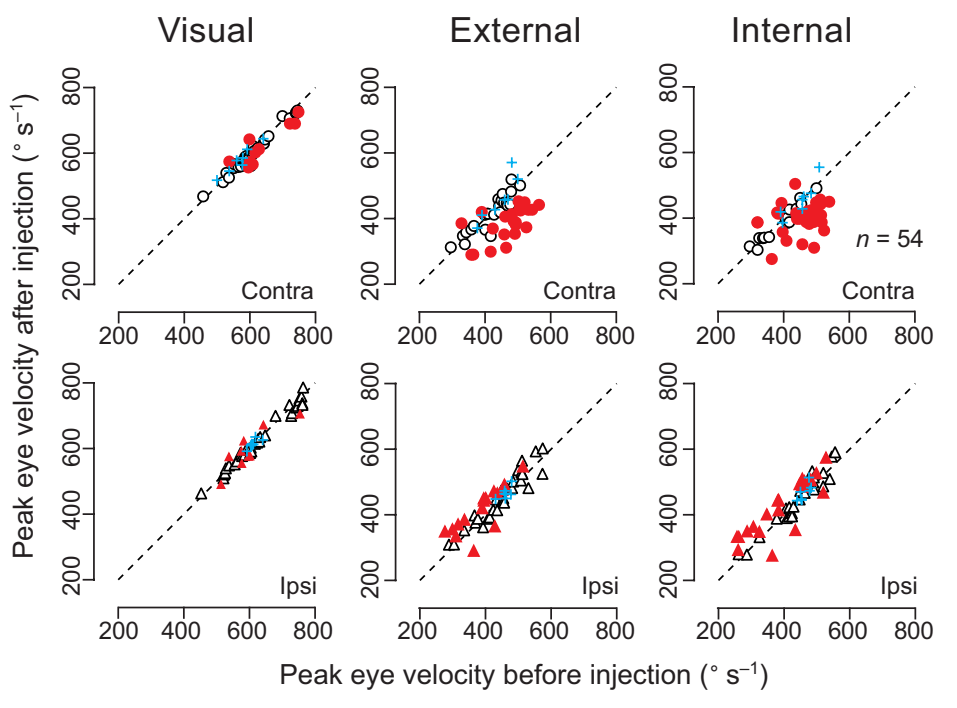

Supplementary Figure 4. Effects of inactivation on saccade velocity. The graph format is same as for Fig. 3a and Supplementary Figs. 2 and 3. The red symbols plot data showing a significant inactivation effect (two tailed t-test, $P<0.01$ ), whereas the open symbols indicate data without it. Blue crosses indicate data from saline control experiments. In the population, thalamic inactivation significantly altered velocity of contraversive memory-guided saccades (two-tailed t-test, $P<$ $0.01, n=47)$, but not in other four tasks. Injection of saline into the effective sites did not alter saccade velocity in all six paradigms $(P>0.5, n=7)$. The changes in saccade velocity following inactivation averaged $-9.4 \pm 17.8,-42.7 \pm 55.8$ and $-38.3 \pm 58.4^{\circ} \mathrm{s}^{-1}$ for contraversive saccades, and $-2.6 \pm 17.4,13.7 \pm 32.7$ and $16.6 \pm 36.7^{\circ} \mathrm{s}^{-1}$ for ipsiversive saccades. The amount of changes in contraversive saccade velocity was statistically different across paradigms (one-way ANOVA, $P<$ 0.01). A post-hoc comparison (Scheffe) detected a significant difference $(P<0.01)$ between the visually-guided saccades and externally triggered memory-guided saccades, but not for the other two pairs. 


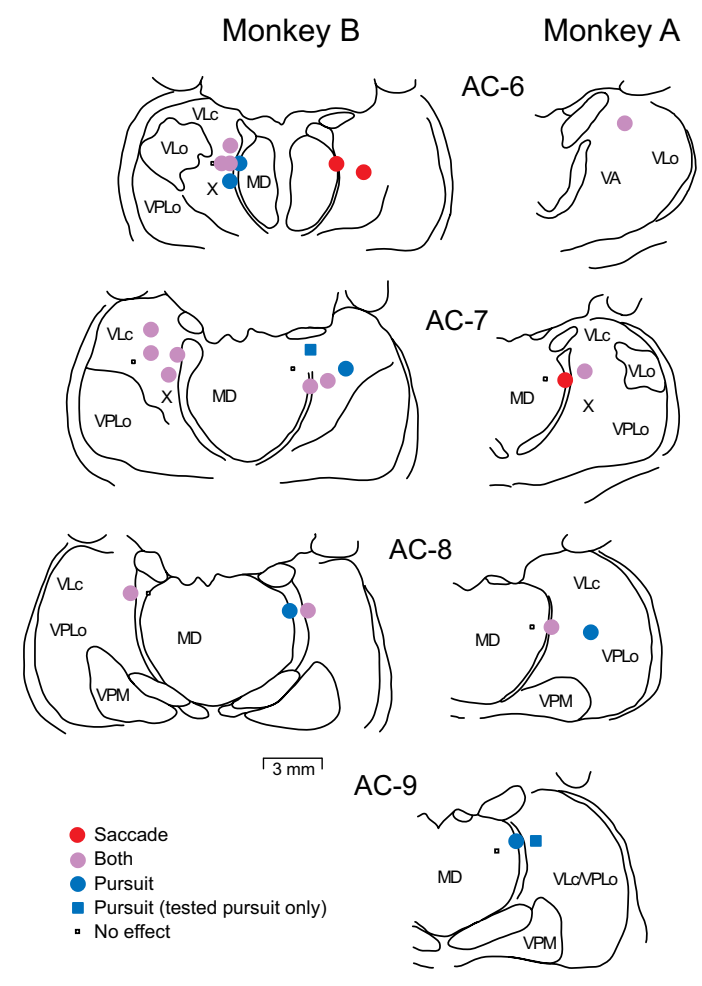

Supplementary Figure 5. Comparison of injection sites that affected saccades or smooth pursuit. Symbols in different colors indicate different effects on eye movements. Although inactivation at many sites altered both types of eye movements, there were some sites where inactivation modified either saccades or pursuit exclusively. 